\title{
AN EVALUATION OF WEIGHTED POLYNOMIAL INTERPOLATION WITH CERTAIN CONDITIONS ON THE ROOTS OF HERMITE POLYNOMIAL
}

\author{
Dhananjay OJHA $^{* 1}$ and R. SRIVASTAVA ${ }^{2}$
}

\begin{abstract}
The purpose of this paper is to construct a polynomial $R_{n}$ of degree at most $3 n-2$ satisfying weighted $(0,2 ; 0)$ interpolation under certain conditions at the zeroes of $H_{n}$ and $H_{n}^{\prime}$, where $H_{n}$ stands for Hermite polynomial. Furthermore, we prove a convergence theorem for $R_{n}$.

2000 Mathematics Subject Classification: 41A10, 33C45, 33C52.

Key words: Lagrange interpolation, convergence, Hermite polynomials, explicit representation, estimations.
\end{abstract}

\section{Introduction}

In 1961, J Balázs [1] initiated the study of weighted $(0,2)$ interpolation on the zeroes of $n^{\text {th }}$ ultraspherical polynomials. L. Szili [14] extended his study by taking roots as the zeroes of $n^{\text {th }}$ Hermite polyomials. In 1975, L.G. Pál [8] introduced a modification of Hermite-Fejér interpolation in which the function values and first derivatives were prescribed on two set of nodes $\left\{x_{k}\right\}_{k=1}^{n}$ and $\left\{y_{k}\right\}_{k=1}^{n}$. He considered they are distributed on the real line such that

$$
-\infty<x_{1}<y_{1}<x_{2}<\ldots .<x_{k}<y_{k} \ldots<y_{n-1}<x_{n}<+\infty
$$

He proved that, there exists a unique polynomial $P_{n}(x)$ of degree at most $2 n-1$ satisfying the following condition:

$$
\left\{\begin{array}{r}
P_{n}\left(x_{k}\right)=\alpha_{k} \\
P_{n}^{\prime}\left(y_{k}\right)=\beta_{k}
\end{array} \quad(k=1,2, \ldots, n-1),\right.
$$

\footnotetext{
1* Corresponding author, Department of Mathematics and Astronomy, Lucknow University, India, e-mail: dhananjaymath2@gmail.com

${ }^{2}$ Department of Mathematics and Astronomy, Lucknow University, India, e-mail: rekhasrivastava4796@gmail.com
} 
with initial condition $P_{n}\left(x_{0}\right)=0$, where $x_{0}$ is a given point different from the nodal points and $\left\{\alpha_{k}\right\}_{k=1}^{n},\left\{\beta_{k}\right\}_{k=1}^{n-1}$ are arbitrary numbers. Later, S.A. Eneduanya [2] proved the convergence for $P_{n}(x)$ on the roots of $\pi_{n}(x)$.

In 1985, L. Szili [15] applied this interpolation process by taking the mixed zeroes of $H_{n}(x)$ and its derivative on infinite interval. For $n$ even, he showed that there exists a uniquely determined polynomial $P_{n}^{*}(x)$ of degree $\leq 2 n-1$, satisfying the conditions:

$$
\begin{aligned}
& \left\{\begin{array}{l}
P_{n}^{*}\left(x_{k}\right)=\alpha_{k}^{*} \quad(k=1,2, \ldots, n), \\
P_{n}^{* \prime}\left(y_{k}\right)=\beta_{k}^{*} \quad(k=1,2, \ldots, n-1),
\end{array}\right. \\
& P_{n}^{*}(0)=-2 \sum_{i=0}^{n} \alpha_{k}^{*}\left[\frac{H_{n}(o)}{H_{n}^{\prime}\left(x_{k}\right)}\right]^{2}
\end{aligned}
$$

which is given by

$$
P_{n}^{*}(x)=\sum_{i=1}^{n} \alpha_{k}^{*} A_{k}(x)+\sum_{i=1}^{n-1} \beta_{k}^{*} B_{k}(x)
$$

and uniqueness does not hold for taking $n$ odd. Furthermore, he proved the convergence theorem for $P_{n}^{*}(x)$. In 1994, I. Joó [5] improved Szili [14] result by modifying the estimate of the fundamental polynomials.

In 1999, Z.F. Sebestyen [9] improved the result of L. Szili [14] and I. Zoo[5] by replacing the condition with an interpolatory condition $P_{n}^{*}(0)=\alpha_{0}$ for $n$ even, where $\alpha_{0}$ is an arbitrary number.

Srivastava and Mathur [12], studied mixed type weighted $(0 ; 0,2)$ interpolation on the mixed zeroes of $H_{n}(x)$ and its derivative which means to determine a polynomial $R_{n}^{*}(x)$ of degree at most $3 n-2$ satisfies the following conditions:

$$
\left\{\begin{aligned}
P_{n}^{* *}\left(x_{k}\right) & =\alpha_{k}^{* *} & & (k=1 \ldots . n), \\
P_{n}^{* *}\left(y_{k}\right) & =\beta_{k}^{* *} & & (k=1, .2, \ldots, n-1), \\
\left(e^{\frac{-x^{2}}{2}} P_{n}^{* *}\right)^{\prime \prime}\left(y_{k}\right) & =\gamma_{k}^{* *} & & (k=1,2, \ldots, n-1),
\end{aligned}\right.
$$

and

$$
P_{n}^{* *}(0)=\sum_{i=0}^{n} \alpha_{k}^{* *} \frac{H_{n}^{\prime \prime}(o) l_{k}^{2}(0)}{H_{n}^{\prime}\left(x_{k}\right)} .
$$

For $n$ even, they proved that, there exists a unique polynomial of degree at most $3 n-2$ satisfying (6)-(7) and for $n$ odd, uniqueness does not exist. Furthermore they proved the convergence theorem for $R_{n}^{*}(x)$.

Also, several authors [12], [10], [6] have studied mixed type interpolation with different conditions on different nodes.

In this paper, we studying the $(0,2 ; 0)$ - interpolation on the zeroes of $H_{n}(x)$ and its derivative with Z.F Sebestyen's[9] conditions.

We have given the following problem. 


\section{Problem:}

Let $x_{0}=0$ be a real number differing from the interscaled system of nodal points (1) where $\left\{x_{k}\right\}_{k=1}^{n}$ and $\left\{y_{k}\right\}_{k=1}^{n-1}$ are the zeroes of $H_{n}(x)$ and $H_{n}^{\prime}(x)$ respectively. We search for a possible minimal degree polynomial $R_{n}(x)$ which satisfies the following interpolation conditions:

$$
\left\{\begin{array}{rc}
R_{n}\left(x_{k}\right)=g_{k} & (k=0,1 \ldots . n), \\
R_{n}\left(y_{k}\right)=g_{k}^{*} & (k=1, .2, \ldots, n-1), \\
\left(e^{\frac{-x^{2}}{2}} R_{n}\right)^{\prime \prime}\left(y_{k}\right)=g_{k}^{* *} & (k=1,2, \ldots, n-1),
\end{array}\right.
$$

\section{Preliminaries}

In this section, we gave some well-known results, which we will use to prove Theorem 1, Lemma 1, Lemma 2, Lemma 3 and Theorem 2.

The differential equation satisfied by $H_{n}(x)$ is given by

$$
\begin{gathered}
H_{n}^{\prime \prime}(x)-2 x H_{n}^{\prime}(x)+2 n H_{n}(x)=0 \\
H_{n}^{\prime}(x)=2 n H_{n-1}(x)
\end{gathered}
$$

The fundamental polynomials of Lagrange interpolation corresponding to the nodal point $x_{k}$ and $y_{k}$ are given by

$$
\begin{gathered}
l_{k}(x)=\frac{H_{n}(x)}{H_{n}^{\prime}\left(x_{k}\right)\left(x-x_{k}\right)} \quad k=1 \ldots . . n \\
L_{k}(x)=\frac{H_{n}^{\prime}(x)}{H_{n}^{\prime \prime}\left(y_{k}\right)\left(x-y_{k}\right)} \quad k=1 \ldots . n-1
\end{gathered}
$$

and they satisfy the conditions given below

$$
\begin{aligned}
& l_{k}\left(x_{j}\right)=\left\{\begin{array}{ll}
0 & \text { for } j \neq k \\
1 & \text { for } j=k
\end{array} \quad \text { for } k=1 \ldots . n\right. \\
& L_{k}\left(y_{j}\right)=\left\{\begin{array}{ll}
0 & \text { for } j \neq k \\
1 & \text { for } j=k
\end{array} \quad \text { for } \quad k=1 \ldots . n-1\right. \\
& l_{k}^{\prime}\left(x_{j}\right)=\left\{\begin{aligned}
\frac{H_{n}^{\prime}\left(x_{j}\right)}{H_{n}\left(x_{k}\right)\left(x_{j}-x_{k}\right)} & \text { for } j \neq k \\
x_{k} & \text { for } j=k
\end{aligned} \quad \text { for } k=1 \ldots n\right. \\
& L_{k}^{\prime}\left(y_{j}\right)=\left\{\begin{aligned}
\frac{H_{n}^{\prime \prime}\left(y_{j}\right)}{H_{n}^{\prime \prime}\left(y_{k}\right)\left(x_{j}-x_{k}\right)} & \text { for } j \neq k \\
y_{k} & \text { for } j=k
\end{aligned} \quad \text { for } k=1 \ldots . . n-1\right. \\
& L_{k}^{\prime \prime}\left(y_{j}\right)=\left\{\begin{aligned}
\frac{2 H_{n}^{\prime \prime}\left(y_{j}\right)}{H_{n}^{\prime \prime}\left(y_{k}\right)\left(x_{j}-x_{k}\right)}\left\{y_{j}-\frac{1}{y_{j}-y_{k}}\right\} & \text { for } j \neq k \\
\frac{4 y_{k}^{2}-2(n-2)}{3} & \text { for } j=k
\end{aligned} \text { for } k=1 \ldots . n\right.
\end{aligned}
$$


G. Szegö, [13] gave following results:

For the roots of $H_{n}(x)$, we have

$$
\begin{gathered}
x_{k}^{2} \sim \frac{k^{2}}{n} \\
H_{n}(x)=O\left(n^{\frac{1}{4}} \sqrt{2^{n} n !}(1+\sqrt[3]{|x|}) e^{\frac{x_{k}^{2}}{2}}\right) \quad x \in R \\
\left|l_{k}(x)\right|=O(1) \frac{2^{n+1} n ! \sqrt{n} e^{\frac{\nu\left(x^{2}+x_{k}^{2}\right)}{2}}}{H_{n}^{\prime 2}\left(x_{k}\right)} \nu>1 \text { and } k=1 \ldots n
\end{gathered}
$$

R. Srivastava and K.K Mathur [11], proved that

$$
\left|L_{k}(x)\right|=O\left(\frac{2^{n} n ! e^{\frac{\nu\left(x^{2}+y_{k}^{2}\right)}{2}}}{\sqrt{n} H_{n}^{2}\left(y_{k}\right)}\right) \quad \nu>1 \text { and } k=1 \ldots n-1
$$

L. Szili [15] gave following results

$$
\begin{gathered}
\left|H_{n}^{\prime}\left(x_{k}\right)\right| \geq c_{1} n^{\frac{1}{4} \sqrt{2^{n+1} n !}} e^{\frac{\delta x_{k}^{2}}{2}} \quad(i=1 \ldots . . n) \\
\left|H_{n}\left(y_{k}\right)\right| \geq c_{2} n^{\frac{-1}{4}} \sqrt{2^{n+1} n !} e^{\frac{\delta y_{k}^{2}}{2}} \quad(i=1 \ldots . n-1),
\end{gathered}
$$

where $c_{1}, c_{2}$ are constants which are independent of $n$ and $0<\delta<1$ is an arbitrarily given real number. He also proved that

$$
\begin{gathered}
\sum_{i=0}^{n} e^{-\epsilon x_{k}^{2}}=O(\sqrt{n}) \\
\sum_{i=0}^{n} \frac{e^{\delta x_{k}^{2}}}{H_{n}^{\prime 2}\left(x_{k}\right)}=O\left(2^{n+1} n !\right)^{-1}
\end{gathered}
$$

Definition: $\omega(f, \delta)$ denotes the special form of modulus of continuity introduced by G.Freud [4], given by

$$
\omega(f, \delta)=\sup _{0 \leq t \leq \delta}\{\|W(x+t) f(x+t)-W(x) f(x)\|+\|\tau(\delta x) W(x) f(x)\|\},
$$

where

$$
\tau(x)=\left\{\begin{array}{rrr}
|x| & \text { for } & |x| \leq 1 \\
1 & \text { for } & |x|>1
\end{array}\right.
$$

and $\|\cdot\|$ denotes the sup-norm in $C(\mathbb{R})$, if $f \in C(\mathbb{R})$ and $\lim _{|x| \rightarrow \infty} W(x) f(x)=0$ then $\lim _{\delta \rightarrow 0} \omega(f, \delta)=0$.

G.Freud[3](Theorem 4) and Theorem 1[1] gave the following results: 
Let $f: \mathbb{R} \rightarrow \mathbb{R}$ be continuously differentiable. Further, let

$$
\left\{\begin{aligned}
\lim _{|x| \rightarrow+\infty} x^{2 k} f(x) e^{\frac{-x^{2}}{2}} & =0 \quad(k=0,1, \ldots) \quad x \in \mathbb{R} \\
\text { and } \lim _{|x| \rightarrow+\infty} f^{\prime}(x) e^{\frac{-x^{2}}{2}}=0 \quad x \in \mathbb{R} &
\end{aligned}\right.
$$

then there exists a polynomial $Q_{n}(x)$ of degree $\leq n$ such that

$$
\begin{gathered}
e^{\frac{-x^{2}}{2}}\left|f(x)-Q_{n}(x)\right|=O\left(\frac{1}{\sqrt{n}}\right) \omega\left(f^{\prime} ; \frac{1}{\sqrt{n}}\right) \\
e^{\frac{-x^{2}}{2}}\left|f^{\prime}(x)-Q_{n}^{\prime}(x)\right|=O(1) \omega\left(f^{\prime} ; \frac{1}{\sqrt{n}}\right)
\end{gathered}
$$

where $\omega$ stands for modulus of continuity defined by (26).

Szili[14]( Lemma 4, Theorem 4) established the following. For $x \in R$

$$
\begin{aligned}
& e^{\frac{-x^{2}}{2}}\left|Q_{n}(x)\right|=O(1) \\
& e^{\frac{-x^{2}}{2}}\left|Q_{n}^{\prime}(x)\right|=O(1)
\end{aligned}
$$

and

$$
e^{\frac{-x^{2}}{2}}\left|Q_{n}^{\prime \prime}(x)\right|=O(1) \sqrt{n} \omega\left(f^{\prime} ; \frac{1}{\sqrt{n}}\right), \text { for }|x|<\sqrt{2 n+1}
$$

\section{Explicit representation of interpolatory polynomial}

In this section, we have proved explicit representation of fundamental polynomials.

Theorem 1. There exists a polynomial

$$
R_{n}(x)=\sum_{k=0}^{n} g_{k} A_{k}(x)+\sum_{k=1}^{n-1} g_{k}^{*} B_{k}(x)+\sum_{k=1}^{n-1} g_{k}^{* *} C_{k}(x)
$$

of degree 3n-2 satisfying condition (8), where $A_{k}(x)(k=0,1,2 \ldots, n)$ and $B_{k}(x)$ $(k=1,2 \ldots ., n-1)$ are the fundamental polynomial of first kind and $C_{k}(x)(k=$ $1,2 \ldots ., n-1)$ are fundamental polynomials of second kind of weighted $(0,2 ; 0)$ interpolation. Each such fundamental polynomials of degree at most $3 n-2$ is given by

$$
\begin{gathered}
A_{0}(x)=\frac{H_{n}{ }^{\prime}(x) H_{n}(x)}{H_{n}{ }^{\prime}(0) H_{n}(0)} \\
A_{k}(x)=\frac{x^{n} H_{n}{ }^{\prime}(x) l_{k}(x)}{x_{k}^{n} H_{n}{ }^{\prime}\left(x_{k}\right)}+\frac{2 H_{n}(x) H_{n}^{\prime}(x)}{x_{k}^{n}\left(H_{n}^{\prime}\left(x_{k}\right)\right)^{2}} \int_{0}^{x} \frac{(n+1) t^{n}-n t^{n-1} x_{k}}{\left(t-y_{k}\right)^{2}} d t
\end{gathered}
$$




$$
\begin{gathered}
B_{k}(x)=\frac{x^{n} H_{n}(x) L_{k}(x)}{y_{k}^{n} H_{n}\left(y_{k}\right)}+\frac{H_{n}(x) H_{n}^{\prime}(x)}{n y_{k}^{n} H_{n}^{2}\left(y_{k}\right)} \int_{0}^{x} \frac{(n-1) t^{n}-y_{k} t^{n-1}}{\left(t-y_{k}\right)^{2}} d t \\
-e^{\frac{-y_{k}^{2}}{2}}\left(y_{k}^{2}+(n-1) y_{k}^{-2}-(3 n+2)\right) C_{k}(x) \\
C_{k}(x)=-\frac{H_{n}(x) H_{n}{ }^{\prime}(x) e^{\frac{y_{k}^{2}}{2}}}{4 n H_{n}^{2}\left(y_{k}\right)} \int_{0}^{x} L_{k}(t) d t
\end{gathered}
$$

Proof. It is enough to show that the polynomials $A_{k}(x)(k=0,1,2 \ldots, n), B_{k}(x)$ $(k=1,2 \ldots, n-1)$, and $C_{k}(x)(k=1,2 \ldots, n-1)$ have the following properties:

$$
\begin{aligned}
& A_{k}\left(x_{j}\right)=\left\{\begin{array}{ll}
0 & \text { for } j \neq k \\
1 & \text { for } j=k
\end{array} \quad \text { for }(j, k=0, \ldots, n),\right. \\
& A_{k}\left(y_{j}\right)=0 \quad(j=1 \ldots n-1, k=0, \ldots, n), \\
& \left(e^{\frac{-x^{2}}{2}} A_{k}\right)^{\prime \prime}\left(y_{j}\right)=0 \quad(j=1, \ldots n-1, k=0, \ldots, n) \\
& B_{k}\left(y_{j}\right)=\left\{\begin{array}{ll}
0 & \text { for } j \neq k \\
1 & \text { for } j=k
\end{array} \quad \text { for } \quad(j, k=1, \ldots, n-1)\right. \text {, } \\
& B_{k}\left(x_{j}\right)=0 \quad(j=0, . . n, k=1, . ., n-1), \\
& \left(e^{\frac{-x^{2}}{2}} B_{k}\right)^{\prime \prime}\left(y_{j}\right)=0 \quad(j, k=1, \ldots, n-1)
\end{aligned}
$$

and

$$
\begin{gathered}
\left(e^{\frac{-x^{2}}{2}} C_{k}\right)^{\prime \prime}\left(y_{j}\right)=\left\{\begin{array}{ll}
0 & \text { for } j \neq k \\
1 & \text { for } j=k
\end{array} \text { for }(j, k=1, \ldots, n-1),\right. \\
C_{k}\left(y_{j}\right)=0, \quad(j, k=0, \ldots, n-1), \\
C_{k}\left(x_{j}\right)=0 \quad(j=0, \ldots n, k=1, . ., n-1) .
\end{gathered}
$$

First, we construct the polynomials $C_{k}(x)$. Let $\mathrm{k}$ be fixed $(\mathrm{k} \in\{1, \ldots, \mathrm{n}-1\})$, from (40) it follows that

$$
C_{k}(x)=H_{n}(x) H_{n}{ }^{\prime}(x) q_{k}(x),
$$

where $p_{k}(x)$ is the polynomial such that,

$$
p_{k}(0)=0
$$

By (41), we get

$$
\left(e^{\frac{-x^{2}}{2}} C_{k}\right)^{\prime \prime}\left(y_{j}\right)=4 n e^{\frac{-y_{k}^{2}}{2}} H_{n}^{2}\left(y_{j}\right) q_{k}^{\prime}\left(y_{j}\right),
$$

(43) satisfies (40), only if,

$$
q_{k}(x)=\frac{1}{4 n e^{\frac{-y_{k}^{2}}{2}} H_{n}^{2}\left(y_{j}\right) q_{k}^{\prime}\left(y_{j}\right)} \int_{0}^{x} L_{k}(t) d t .
$$

Combining (44), (41), we obtain (37). Obviously, $C_{k}(x)$ is a polynomial of degree $3 n-2$, which satisfies (40). Second, we construct $B_{k}(x), \mathrm{k}$ be fixed $(\mathrm{k} \in\{1, \ldots \mathrm{n}-1\})$. We look for $B_{k}(x)$ in the following form

$$
B_{k}(x)=c_{1} x^{n} H_{n} L_{k}(x)+H_{n}(x) H_{n}^{\prime}(x) w_{k}(x)+c_{2} . C_{k}(x)
$$


where $w_{k}(x)$ is the suitable polynomial for which

$$
w_{k}(0)=0
$$

and $c_{1}, c_{2}$ are arbitrary constants. According to (39) $q_{k}(x)$, for $\mathrm{j} \neq \mathrm{k}$

$$
B_{k}\left(y_{j}\right)=0
$$

and for $j=k$

$$
B_{k}\left(y_{k}\right)=1 \Longrightarrow c_{1}=\frac{1}{y_{k}^{n} H_{n}\left(y_{k}\right)}
$$

from (45) and (39) we get for $j \neq k$

$$
\begin{aligned}
& \left(e^{\frac{-x^{2}}{2}} B_{k}\right)^{\prime \prime}\left(y_{j}\right) \\
= & \frac{2 e^{\frac{-y_{j}^{2}}{2}} H_{n}^{2}\left(y_{j}\right)}{H_{n}\left(y_{k}\right)}\left[\frac{(n-1) y_{j}^{n}-y_{k} y_{j}^{n-1}}{\left(y_{j}-y_{k}\right)^{2}}\right]-2 n e^{\frac{-y_{j}^{2}}{2}} H_{n}^{2}\left(y_{j}\right) w_{k}^{\prime}(x)=0
\end{aligned}
$$

for $j=k$

$$
\left(e^{\frac{-x^{2}}{2}} B_{k}\right)^{\prime \prime}\left(y_{k}\right)=e^{\frac{-y_{k}^{2}}{2}}\left(y_{k}^{2}+(n-1)-(3 n-2)\right)+c_{2}=0
$$

From (49) and (50), we conclude that

$$
w_{k}(x)=\frac{1}{n} \int_{0}^{x} \frac{(n-1) t^{n}-y_{k} t^{n-1}}{\left(t-y_{k}\right)^{2}} d t
$$

and

$$
c_{2}=-e^{\frac{-y_{k}^{2}}{2}}\left(y_{k}^{2}+(n-1) y_{k}^{-2}-(3 n-2)\right)
$$

Combining (48), (51) and (52), we get (36). It is easy to see that, $B_{k}(x)$ is a polynomial of degree $3 n-2$, which satisfies (39).

Proof of $A_{k}(x)$ is like proof of $B_{k}(x)$.

\section{Order of convergence of fundamental polynomials}

In this Section, we the compute order of convergence of fundamental polynomials, which is required to prove theorem 2

Lemma 1. For $k=0,1 \ldots n$ and $x \in(-\infty,+\infty)$

$$
\sum_{i=0}^{n} e^{\beta x_{k}^{2}}\left|A_{k}(x)\right|=O(\sqrt{n} \log n) e^{\nu x^{2}} \quad \text { for } \quad \nu>\frac{3}{2}, 0<\beta<1
$$

where $A_{k}(x)$ is given by (35) 
Proof. From (35) we have

$$
\begin{aligned}
\sum_{k=0}^{n} e^{\beta x_{k}^{2}}\left|A_{k}(x)\right| \leq & \sum_{k=0}^{n} e^{\beta x_{k}^{2}} \frac{\left|x^{n}\right|\left|H_{n}^{\prime}(x)\right|\left|l_{k}(x)\right|}{\left|x_{k}^{n}\right|\left|H_{n}^{\prime}\left(x_{k}\right)\right|} \\
& +\sum_{k=0}^{n} e^{\beta x_{k}^{2}} \frac{2\left|H_{n}(x)\right|\left|H_{n}^{\prime}(x)\right|}{\left|x_{k}^{n}\right|\left|\left(H_{n}^{\prime}\left(x_{k}\right)\right)^{2}\right|}\left|\int_{0}^{x} \frac{(n+1) t^{n}-n t^{n-1} x_{k}}{\left(t-y_{k}\right)^{2}} d t\right| \\
\leq & \zeta_{1}+\zeta_{2}
\end{aligned}
$$

using (10), (18), (19), (20), (22) and (24), we have

$$
\zeta_{1}=\sum_{k=0}^{n} e^{\beta x_{k}^{2}} \frac{2 n\left|x^{n}\right|\left|H_{n-1}(x)\right|\left|l_{k}(x)\right|}{\left|x_{k}^{n}\right|\left|H_{n}{ }^{\prime}\left(x_{k}\right)\right|}=O(\sqrt{n}) e^{\nu x^{2}} \text { for } \nu>\frac{3}{2}
$$

Using (10), (18) (19), and (25), we have

$$
\zeta_{2}=O(\log n) \sum_{k=0}^{n} e^{\beta x_{k}^{2}} \frac{4 n\left|H_{n}(x)\right|\left|H_{n-1}(x)\right|}{\left|x_{k}^{n}\right|\left|\left(H_{n}^{\prime}\left(x_{k}\right)\right)^{2}\right|}=O(\sqrt{n} \log n) e^{\nu x^{2}} \text { for } \nu>\frac{3}{2}
$$

Thus, by using (55) and (56) in (54), we get the required lemma.

Lemma 2. For $k=1 \ldots n-1$ and $x \in(-\infty,+\infty)$

$$
\sum_{k=1}^{n-1} e^{\beta y_{k}^{2}}\left|B_{k}(x)\right|=O(\sqrt{n} \log n) e^{\nu x^{2}} \quad \text { where } \quad \nu>\frac{3}{2}, 0<\beta<1
$$

where $B_{k}(x)$ is given by (36)

Proof. From (36), we have

$$
\begin{aligned}
\sum_{k=1}^{n-1} e^{\beta y_{k}^{2}}\left|B_{k}(x)\right| \leq & \sum_{k=1}^{n-1} e^{\beta y_{k}^{2}} \frac{\left|x^{n}\right|\left|H_{n}(x)\right|\left|L_{k}(x)\right|}{\left|y_{k}^{n}\right|\left|H_{n}\left(y_{k}\right)\right|} \\
& -\sum_{k=1}^{n-1} e^{\beta y_{k}^{2}} \frac{\left|H_{n}(x)\right|\left|H_{n}^{\prime}(x)\right|}{n\left|y_{k}^{n}\right|\left|H_{n}^{2}\left(y_{k}\right)\right|}\left|\int_{0}^{x} \frac{(n-1) t^{n}-y_{k} t^{n-1}}{\left(t-y_{k}\right)^{2}} d t\right| \\
& +\sum_{k=1}^{n-1} e^{\beta y_{k}^{2}}\left|\left(y_{k}^{2}+(n-1) y_{k}^{-2}-(3 n+2)\right)\right|\left|C_{k}(x)\right| \\
\leq & \zeta_{1}+\zeta_{2}+\zeta_{3}
\end{aligned}
$$

By using (10), (19), (21), (23) and (24), we have

$$
\zeta_{1}=O(\sqrt{n}) e^{\nu x^{2}} \text { for } \nu>\frac{3}{2}
$$

By using (10), (18) (19), (24), we have

$$
\zeta_{2}=O(\log n) \sum_{k=0}^{n} e^{\beta x_{k}^{2}} \frac{2\left|H_{n}(x)\right|\left|H_{n-1}(x)\right|}{\left|y_{k}^{n}\right|\left|H_{n}^{2}\left(y_{k}\right)\right|}=O(\sqrt{n} \log n) e^{\nu x^{2}} \text { for } \nu>\frac{3}{2}
$$


By using (18), (26) and [11](lemma 5.3), we have

$$
\zeta_{3}=O(\sqrt{n} \log n) e^{\nu x^{2}} \text { for } \nu>\frac{3}{2}
$$

Thus, by using (59)-(61) in (58), lemma follows.

Lemma 3. For $k=1,2, \ldots, n-1$ and $x \in(-\infty,+\infty)$

$$
\sum_{k=1}^{n} e^{\beta y_{k}^{2}}\left|C_{k}(x)\right|=O\left(\frac{\log n}{\sqrt{n}}\right) e^{\nu x^{2}} \quad \text { where } \quad \nu>\frac{3}{2}, 0<\beta<1
$$

where $C_{k}(x)$ is given by (37)

Proof. Lemma follows from[11](lemma 5.3)

\section{Main result: Convergence theorem of interpolatory polynomial}

In this section, we have proved convergence theorem for interpolatory polynomial $R_{n}(x)$.

Theorem 2. Let the interpolated function $f: R \longrightarrow R$ be continuously differentiable such that

$$
\left\{\begin{aligned}
\lim _{|x| \rightarrow+\infty} x^{2 k} f(x) \rho(x)=0 & (k=0,1, \ldots) \\
\lim _{|x| \rightarrow+\infty} f^{\prime}(x) \rho(x)=0 & \text {,where } \quad \rho(x)=e^{\frac{-x^{2}}{2}}
\end{aligned}\right.
$$

further taking the number $\delta_{k}$ such that

$$
\delta_{k}=O\left(\sqrt{n} e^{\delta y_{k}^{2} \omega}\left(f^{\prime} ; \frac{1}{\sqrt{n}}\right)\right), k=1, \ldots, n-1
$$

where $\omega$ is modulus of continuity of $f^{\prime}$. Then

$$
R_{n}(f, x)=\sum_{k=0}^{n} f\left(x_{k}\right) A_{k}(x)+\sum_{k=1}^{n-1} f\left(y_{k}\right) B_{k}(x)+\sum_{k=1}^{n-1} \delta_{k} C_{k}(x)
$$

satisfies the relation

$$
e^{-\nu x^{2}}\left|f(x)-R_{n}(x)\right|=O(\log n) \omega\left(f ; \frac{1}{\sqrt{n}}\right), \nu>\frac{3}{2}
$$

Proof. Since $R_{n}(x)$ given by (33) is exact for all polynomials $Q_{n}(x)$ of degree $\leq$ $3 \mathrm{n}-2$, we have

$$
Q_{n}(x)=\sum_{k=0}^{n} Q_{n}\left(x_{k}\right) A_{k}(x)+\sum_{k=1}^{n-1} Q_{n}\left(y_{k}\right) B_{k}(x)+\sum_{k=1}^{n-1}\left(e^{\frac{-x^{2}}{2}} Q_{n}\right)^{\prime \prime}\left(y_{k}\right) C_{k}(x)
$$


Using lemma (1)-(2), (65), (67) (26) and (28)-(31), it can be easily seen that,

$$
\begin{aligned}
e^{-\nu x^{2}}\left|R_{n}(x)-f(x)\right| \leq & e^{-\nu x^{2}}\left|R_{n}(x)-f(x)\right|+e^{-\nu x^{2}} \sum_{k=0}^{n}\left|f\left(x_{k}\right)-Q_{n}\left(x_{k}\right)\right|\left|A_{k}(x)\right| \\
& +e^{-\nu x^{2}} \sum_{k=1}^{n-1}\left|f\left(y_{k}\right)-Q_{n}\left(y_{k}\right)\right|\left|B_{k}(x)\right| \\
& +e^{-\nu x^{2}} \sum_{k=1}^{n-1}\left|\left(e^{\frac{-x^{2}}{2}} Q_{n}\right)^{\prime \prime}\left(y_{k}\right)-\delta_{k}\right|\left|C_{k}(x)\right| \\
\leq & O(1) \omega\left(f^{\prime} ; \frac{1}{\sqrt{n}}\right)+O(\log n) \omega\left(f^{\prime} ; \frac{1}{\sqrt{n}}\right) \\
& +e^{-\nu x^{2}} \sum_{k=1}^{n-1}\left|e^{\frac{-y_{k}^{2}}{2}} Q_{n}^{\prime \prime}\left(y_{k}\right)\right|\left|C_{k}(x)\right| \\
& +e^{-\nu x^{2}} \sum_{k=1}^{n-1}\left|\left(e^{\frac{-y_{k}^{2}}{2}}\right)^{\prime} Q_{n}^{\prime}\left(y_{k}\right)\right|\left|C_{k}(x)\right| \\
& +e^{-\nu x^{2}} \sum_{k=1}^{n-1}\left|\left(e^{\frac{-y_{k}^{2}}{2}}\right)^{\prime \prime} Q_{n}\left(y_{k}\right)\right|\left|C_{k}(x)\right| \\
& +e^{-\nu x^{2}} \sum_{k=1}^{n-1}\left|\delta_{k} C_{k}(x)\right|
\end{aligned}
$$

Thus by using lemma (3), (30)-(32) and (64) in (68), we get the proof of the required theorem.

\section{Conclusion:}

Let $\left\{x_{k}\right\}_{k=1}^{n}$ and $\left\{y_{k}\right\}_{k=1}^{n-1}$ be the roots of Hermite polynomial $H_{n}(x)$ and its derivative $H_{n}{ }^{\prime}(x)$ respectively. If $\mathrm{f}(\mathrm{x})$ is a continuously differentiable function on $(-\infty,+\infty)$ satisfying (63), then their exist a polynomial $R_{n}(x)(33)$ satisfying condition (8), which uniformly converges to $\mathrm{f}(\mathrm{x})$ on $(-\infty,+\infty)$ as $n \rightarrow \infty$.

\section{References}

[1] Balázs, J., Sülyosott (0,2)-interpoláció ultraszférikus polinomk gyökein, MTA III Oszt. Közl 11 (1961), 305-338.

[2] Eneduanya, S.A. On the convergence of interpolation polynomials, Anal. Math. 11 (1985), 13-22.

[3] Freud, G., On the convergence of Lagrange interpolation process on infinite intervals. Mat. Lapok., 18 (1967), 289-292. 
Weighted polynomial interpolation

[4] Freud, G., On two polynomial inequalities I, Acta Math. Acad. Sci. Hung., 22 (1972), 137-145

[5] Joó, I., On Pál interpolation, Ann. Univ. Sci. Budapest. Eötvös Sect. Math. 37 (1994), 247-262.

[6] Mathur, P. and Dutta, S., On pal type Weighted lacunary $(0,2 ; 0)$ - interpolation on infinite interval $(-\infty,+\infty)$, Approximation Theory Appl. 17 (2001), no. $4,1-10$.

[7] Mathur, K.K. and Sharma, A., Some interpolatory properties of Hermite polynomials, Acta Math. Acad. Sci. Hungar. 12 (1961), 193-207.

[8] Pál, L.G., A new modification of the Hermite-Fejér interpolation, Anal. Math. 1 (1975), 197-205.

[9] Sebestyń, Z.F, Supplement to the Pál type $(0 ; 0,1)$ lacunary interpolation, Anal. Math. 25 (1999), 147-154.

[10] Singh, Y. and Srivastava, R., An analysis of (0,1:0) interpolation based on the zeros of ultraspherical polynomials, Bull. Transilv. Univ. Braşov, Ser. III 12(61), (2019), no. 1, 95-126.

[11] Srivastava, R. and Mathur, K.K., Weighted (0;0,2)-interpolation on the root of hermite polynomials, Acta Math. Hungar. 70 (1996), no. 1-2, 57-73.

[12] Srivastava, R. and Mathur, K.K., An interpolation process on the roots of Hermite polynomials (0;01)-interpolation on infinite interval, Bull. Inst. Math. Acad. Sin. (N.S.) 26 (1998), 229-237.

[13] Szegö, G., Orthogonal polynomial, Amer. Math. Soc., Coll. Publ., New York, 1959.

[14] Szili, L., Weighted (0,2)-interpolation on the roots of Hermite polynomials, Ann. Univ. Sci. Budapest. Eötvös Sect. Math. 27 (1984), 153-166.

[15] Szili, L., A convergence theorem for the Pál method of interpolation on the roots of Hermite polynomials, Anal. Math. 11 (1985), 75-84. 
